\title{
Investigation of pre-flare role of Ellerman bombs in solar active regions
}

\author{
Yu. V. Papernaya ${ }^{1}$ and L. K. Kashapova ${ }^{2}$ \\ ${ }^{1}$ Physical Department of Irkutsk State University, Irkutsk, Russia \\ email:papernaya@gmail.com \\ ${ }^{2}$ Institute of Solar-Terrestrial Physics, SB RAN, Irkutsk, Russia \\ email: 1kk@iszf.irk.ru
}

\begin{abstract}
Ellerman bombs or moustache are known as short-living fine emission structures associated with a local energy release in solar atmosphere. The relation between the moustaches and flare has been discussed since the moment of the moustaches discovery. The resent results of the Flare Genesis Experiment demonstrated that this problem is still actual and attractive. We took into account the possibility of different mechanisms of their origin (thermal and nonthermal) by using spectro polarimetric data obtained in summer 1999 and carried out analysis of Ellerman bomb's role in flare activity of the Sun by the example of the several active regions.
\end{abstract}

Keywords. solar flare, moustaches, pre-flare activity

\section{Introduction}

Ellerman bombs or moustaches are enigmatic phenomena in the solar atmosphere. The relation between the moustaches and flare has been discussed since the moment of the moustaches discovery (for example, Severny, 1968). The resent results of the Flare Genesis Experiment (Georgoulis et al., 2002; Schmieder et al., 2002) attracted attention to this problem again.

There are the several models suggesting explanation for so fast and at the same time so local energy release. The polarization observations take up the special place among the methods of the moustache investigation. For example, only they allow to extract moustaches which emission were excited by beams of energetic particles. The peculiarity of current study was to choose the moustaches most possibly produced by energetic particle beams. Then we analyzed if something has been changed in flare activity in after "non-thermal" Ellerman bombs observation or not. Obtained results were compared with flare activity evolution after observation of Ellerman bombs which has not indication of non-thermal electron beam presence.

\section{Observational data}

For our investigation we used spectropolarimetric observations of 164 moustaches obtained by Large Solar Vacuum Telescope in summer 1999. The criterion for selection of Ellerman bombs with most probable non-thermal mechanism of origin was a linear polarization in $\mathrm{H} \alpha$ line center. A information about possible mechanism of Ellerman bomb origin was gotten from Zharkova \& Kashapova (2005). Results are presented in Table 1. The flare activity evolution was investigate 7 days before and after moment of moustaches observation. Thus, during this period we take into account all flares ( $\mathrm{H} \alpha$ flares, hard and soft x-ray flares) occurred in active region (AR) where the moustaches were 
Table 1. The time of observation and location of moustaches with significant polarization

\begin{tabular}{ccc}
\hline DATE & AR & UT \\
\hline 22.06 .1999 & 8594 & $5: 49-5: 50$ \\
27.06 .1999 & 8598 & $9: 38$ \\
28.06 .1999 & 8611 & $7: 50-7: 55$ \\
04.07 .1999 & 8614 & $8: 46-9: 26$ \\
14.07 .1999 & 8627 & $7: 10-7: 28$ \\
06.08 .1999 & 8656 & $1: 11-1: 13$ \\
09.08 .1999 & 8662 & $9: 14$ \\
\hline
\end{tabular}

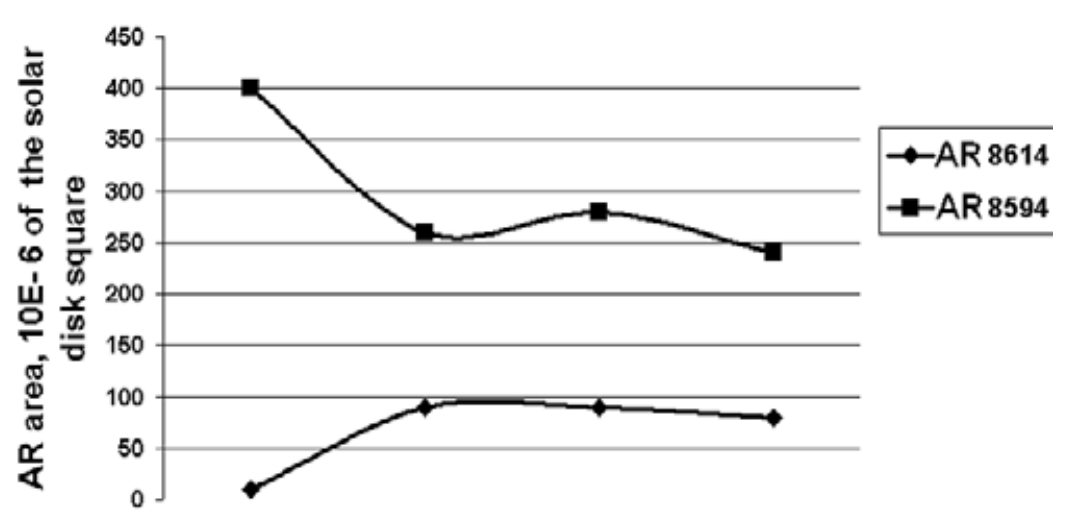

Figure 1. Evolution of AR area during the study period for cases of flare activity increase (AR 8614) and absence of considerable changes (AR 8594).

found. For this purpose we used data from the flare observation catalogues by GOES, Yohkoh and BATSE.

\section{Preliminary conclusions}

- Flare activity increase was not revealed in AR where Ellerman bombs with thermal mechanism excitation were observed.

- A flare activity response was ambiguous in AR where Ellerman bombs with nonthermal mechanism excitation were observed.

- During current study, a rise of flare activity was revealed only for AR with the small (less then 100 millionths of the solar hemisphere) and developing area where Ellerman bombs with non-thermal mechanism excitation were observed (Fig. 1).

\section{Acknowledgements}

We would like to thank Dr. M.A. Livshits for idea of this study. Authors acknowledge the teams of GOES, Yohkoh and BATSE for providing data used in this publication.

\section{References}

Georgoulis, M.K., Rust, D.M., Bernasconi, P.N. \& Schmieder, B. 2002 Astrophysical Journal $\mathbf{5 7 5}, 506-528$.

Severny, A. B. 1968 Proceedings of the 9th Nobel Symposium, 71

Schmieder, B., Pariat, E., Aulanier, G., Georgoulis, M.K., Rust, D.M. \& Bernasconi P.N. 2002 Proc. 10th. European Solar Physics Meeting SP-506, 911

Zharkova, V. V. and Kashapova, L. K. 2003 Astronomy $\&$ Astrophysics 431, 1075 